\title{
Inhibition of GPR4 attenuates SH-SY5Y cell injury in cerebral ischemia/reperfusion via anti-apoptotic pathways
}

\author{
Chunli Xing ${ }^{1}$, Guizhen Yan ${ }^{1}$ and Qishuai Liu ${ }^{2}$ \\ 1Department of Neurology, People's Hospital of Lixia District of Jinan, Jinan City, Shandong Province, 250014, China; ${ }^{2}$ Department of Neurology, \\ The First Hospital of Zibo, Zibo City, Shandong Province, 255200, China
}

Cerebral ischemia/reperfusion injury (CIRI) can lead to increased vascular endothelial permeability and blood-brain barrier damage in patients with stroke. G protein-coupled receptor 4 (GPR4) is a functional pH sensor that plays a key role in renal ischemia-reperfusion-induced apoptosis. However, whether GPR4 has a role in cerebral ischemia remains to be further studied. Our study found that after oxygen-glucose deprivation/reoxygenation (OGD/R) treatment, the levels of GPR4 and CHOP in SH-SY5Y cells were significantly increased, which was accompanied by a decrease in cell viability, and an increase in LDH release and apoptosis. After knockdown of GPR4 using shRNA, CHOP levels in SH-SY5Y cells were also decreased, which unexpectedly increased cell activity and decreased LDH release and apoptosis rate. Interestingly, CHOP overexpression reversed the effect of GPR4 knockdown, suggesting that OGD/R-induced CIRI may involve endoplasmic reticulum stress-related apoptosis. In conclusion, our study provided a basis for further research on the mechanism of CIRI.

Keywords: G protein-coupled receptor 4; Cerebral ischemia/reperfusion injury; oxygen-glucose deprivation/reoxygenation; Apoptosis; SH-SY5Y cell line injury

Received: 23 September, 2020; revised: 09 November, 2020; accepted: 13 November, 2020; available on-line: 07 March, 2021

囚e-mail: yanguizhen1014@163.com

Abbreviations: CIRI, Cerebral ischemia/reperfusion injury; CST, Cell Signaling Technology; CHOP, C/EBP-homologous protein; ECs, endothelial cells; ER, Endoplasmic reticulum; ERS, Endoplasmic reticulum stress; FBS, fetal bovine serum; GPR4, G protein-coupled receptor 4; MTT, 3-(4,5-dimethylthiazol-2-yl)-2,5-diphenyltetrazolium bromide; LDH, Lactate dehydrogenase; OGD/R, oxygen-glucose deprivation/ reoxygenation; shNC, shRNA negative control;

\section{INTRODUCTION}

Cerebral ischemia/reperfusion injury (CIRI) is a condition in which blood perfusion recovers after short-term ischemia and hypoxia, which can aggravate brain tissue damage (Zhao et al., 2018). Oxidative stress, apoptosis and activated inflammation are a series of common reactions after cerebral ischemia-reperfusion injury ( $\mathrm{Hu}$ et al., 2017; Wu et al., 2018). Therefore, inhibition of oxidative stress, apoptosis and activated inflammation is of great significance for the treatment and prognosis of cerebral ischemia-reperfusion injury (Pfeilschifter et al., 2011). However, the mechanism of CIRI causing this series of reactions is complicated and still needs further exploration.

Nuclear transcription factor C/EBP-homologous protein (CHOP) exists widely in mammalian cells, and proteins regulated by $\mathrm{CHOP}$ are related to a variety of cellular activities, such as proliferation (Zhou et al., 2015), differentiation (Shang et al., 2017) and apoptosis (Hu et al., 2018), and it is also a specific transcription factor for endoplasmic reticulum stress. When endoplasmic reticulum stress occurs, apoptosis can be induced by increased expression of CHOP. A study demonstrated that in cortical neuronal injury induced by $\mathrm{OGD} / \mathrm{R}$, the expression of CHOP is up-regulated, indicating that the overexpression of $\mathrm{CHOP}$ is related to CIRI (Zhang et al., 2019).

$\mathrm{G}$ protein-coupled receptor 4 (GPR4) is a functional $\mathrm{pH}$ sensor, which is mainly expressed in endothelial cells (ECs) (Sun et al., 2010). Studies showed that GPR4 activation caused by acidosis is a mediating factor of endoplasmic reticulum stress (Dong et al., 2017). The activation of GPR4 caused by acidosis also increases the expression of some stress response genes, such as CHOP (Dong et al., 2013). Notably, GPR4 plays a key role in HR/acidificationinduced CHOP expression and apoptosis induction (Dong et al., 2014). Recent studies showed that GPR4 has a certain effect on tissue ischemia-reperfusion. The expression of GPR4 increases during renal ischemia-reperfusion. GPR4 gene knockdown can improve renal ischemia-reperfusion injury and decrease cell apoptosis by inhibiting the expression of CHOP (Dong et al., 2017). However, whether GPR4 has a role in cerebral ischemia remains to be further studied.

In the current study, the role of GPR4 in OGD/Rtreated SH-SY5Y cells and its potential molecular mechanisms were investigated in vitro, and our study provided a basis for further exploration of the mechanism of CIRI.

\section{MATERIAL AND METHODS}

Cell culture and oxygen-glucose deprivation/ reoxygenation (OGD/R) treatment

Human-derived neuroblastoma SH-SY5Y cells were purchased from ATCC and cultured in DMEM (Gibco; Thermo Fisher Scientific, Massachusetts, USA) containing 10\% heat-inactivated fetal bovine serum (FBS) and 1\% penicillin/streptomycin (Invitrogen; Thermo Fisher Scientific, Inc.) at $37^{\circ} \mathrm{C}$ in a humidified atmosphere. SH-SY5Y cells were incubated in DMEM medium without glucose and serum for $2,4,6$ and $12 \mathrm{~h}$ at $37^{\circ} \mathrm{C}$ under hypoxic conditions $(1 \%$ $\mathrm{O}_{2}, 95 \% \mathrm{~N}_{2}$ and $5 \% \mathrm{CO}_{2}$ ), followed by rapid reoxygenation $\left(95 \%\right.$ air and $5 \%\left(\mathrm{CO}_{2}\right)$, and then cultured in the conventional medium for $24 \mathrm{~h}$ to establish I/R injury cell model.

\section{Western blotting}

The levels of proteins of interest were detected by Western blotting. Proteins $(20 \mu \mathrm{g})$ resolved in 10\% SDSPAGE gels were transferred onto PVDF membranes. Thereafter, the membranes were probed with primary an- 
tibodies: anti-GPR4 (ab97271, 1:1000, Abcam, Cambridge, UK), anti-CHOP (\#5554, 1:1000, Cell Signaling Technology (CST), Danvers, MA, USA), anti-GAPDH (ab9485, 1:2500, Abcam, Cambridge, UK), anti-cleaved caspase 3 (ab32042, 1:500, Abcam, Cambridge, UK) and anticleaved PARP (\#5625, 1:1000, CST), Danvers, MA, USA) overnight at $4^{\circ} \mathrm{C}$. After that, the membranes probed with primary antibodies were incubated with secondary antibody (Cell Signaling Technology, Massachusetts, USA) for $1 \mathrm{~h}$ at room temperature. Bands were visualized with ECL chemiluminescent detection kit (Solarbio, Beijing, China) and data were analyzed using an ImageQuant 350 analyzer (GE Healthcare, Pennsylvania, USA).

\section{GPR4 knockdown}

GRP4 was knocked down using shRNA. In brief, shgpr4\#1, shgpr4\#2 and shRNA negative control (shNC) were cloned into pRNA-H1.1, an shRNA expression vector, to construct recombinant plasmids (GenScript, Piscataway, NJ, USA). After that, SH-SY5Y cells were transfected with the recombinant plasmids $(2 \mu \mathrm{g})$ using Lipofectamine 2000 (Invitrogen, Carlsbad, CA, USA) for GPR4 silencing (shGPR4\#1 or shGPR4\#2). $24 \mathrm{~h}$ after transfection, the cells were cultured and screened with $400 \mu \mathrm{g} / \mathrm{ml}$ G418. Transfection efficiency was determined by qPCR. shRNAs for GPR4 silencing were designed as two pairs of annealed small interfering RNA (siRNA) fragments. The sequences were as follows:

- shRNA\#1 (i) 5'-AAGGCTAATGCTAGCGTCTTGCCTGTCTC-3' (sense), and 5'-AAGACTGCTAGCTGAACGTACCCTGTCTC-3' (antisense); (ii) 5'-AACCCTAGTCGATCTGACTGACCTGTCTC-3' (sense) and 5'-AACT'TGCTGATGCTCGACCGTCCTGTCTC-3' (antisense);

- shRNA\#2 (i) 5'-AAGGT'TGCTGATCTACGTAGGCCTGTCTC-3' (sense), and 5'-AAGTTCGATGCTAGTGACTGGCCTGTCTC-3' (antisense); (ii) 5'-AACAAGCTGACTAGCTGGTCACCTGTCTC-3' (sense) and 5'-AACCCGTAGTCGTCGTAGCTTCCTGTCTC-3' (antisense).

\section{3-(4,5-dimethylthiazol-2-yl)-2,5-diphenyltetrazolium bromide (MTT) assay}

Cell viability was measured using MT'T assay. SH-SY5Y cells were inoculated into 24 -well plates $\left(1 \times 10^{5}\right.$ cells/well $)$ and after $24 \mathrm{~h}$ subjected to OGD/R for $2,4,6$ and $12 \mathrm{~h}$. Then, MT'T reagent $(50 \mu \mathrm{g} / \mathrm{ml})$ was added for another 6 $\mathrm{h}$ of incubation. Subsequently, the cells were treated with DMSO for $5 \mathrm{~min}$, and then the absorbance at $570 \mathrm{~nm}$ was measured using a microplate reader.

\section{Lactate dehydrogenase (LDH) measurement}

Cytotoxicity was evaluated by the determination of LDH activity. LDH release was measured using cytotoxicity detection kit (Beyotime, Shanghai, China) according to the manufacturer's instructions by measuring the absorbance at $490 \mathrm{~nm}$ on a microplate reader (PerkinElmer, California, USA).

\section{Flow cytometry analysis}

Apoptosis was assessed using flow cytometry. SHSY5Y cells were digested with trypsin and stained with Alexa Fluor ${ }^{\circledR} 488$ dye-labeled anti-BrdU antibody according to the manufacturer's operating procedures (APOBrdUTM TUNEL assay kit; Invitrogen; Thermo Fisher Scientific, Inc.). After that, apoptosis determination was carried out using Cytomics FC 500 flow cytometer (Beckman Coulter, California, USA).

\section{CHOP overexpression}

Adenovirus vectors (OE-CHOP) for overexpression of $\mathrm{CHOP}$ and negative control (OE-NC) were obtained from GenePharma (Shanghai, China). SH-SY5Y cells were cultured with OE-CHOP/OE-NC adenovirus (MOI=100) at $37^{\circ} \mathrm{C}$ for $6 \mathrm{~h}$. Then, the medium was removed and the culture was continued in a fresh medium at $37^{\circ} \mathrm{C}$ for 24 h. Cells with stable and high expression of CHOP were banked.

\section{Statistical analysis}

SPSS 19.0 software (International Business Machines Corporation, Armonk, New York, USA) was applied for statistical analysis. Experimental data are presented as the mean $\pm 182 S$.D. The data between two groups were compared using an unpaired Student's t-test, while the data from multiple groups were compared using a One-Way ANOVA followed by Dunnett's posthoc test. $p<0.05$ indicated a statistically significant difference.

\section{RESULTS}

\section{Nerve injury induced by $O G D / R$ is related to the high expression of GPR4 and CHOP}

Firstly, we investigated the expression of GPR4 and CHOP in SH-SY5Y cells after different treatment time. As shown in Fig. 1, compared to the control group, the protein levels of GPR4 and CHOP in the cells after exposure to $\mathrm{OGD} / \mathrm{R}$ increased rapidly and in a time-de-
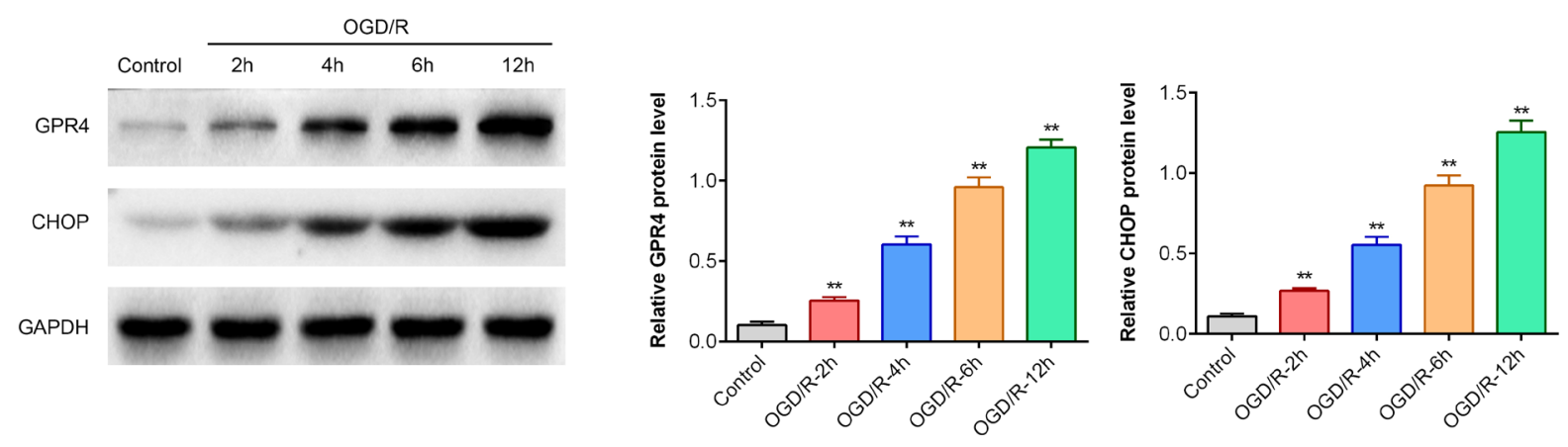

Figure 1. SH-SY5Y cells injury induced by OGD/R is related to the high expression of GPR4 and CHOP.

$\mathrm{SH}-\mathrm{SY} 5 \mathrm{Y}$ cells were cultured in DMEM medium without glucose and serum for $2,4,6$ and $12 \mathrm{~h}$ at $37^{\circ} \mathrm{C}$ under hypoxic conditions (1\% $\mathrm{O}_{2}$, $95 \% \mathrm{~N}_{2}$ and $\left.5 \% \mathrm{CO}_{2}\right)$, followed by rapid reoxygenation $\left(95 \%\right.$ air and $\left.5 \% \mathrm{CO}_{2}\right)$. The protein levels of GPR4 and CHOP were measured by Western blotting. ${ }^{* *} p<0.01$ vs control group. 
A Control OGD/R $\frac{\text { OGD/R }}{\text { ShNC ShGPR4\#1 shGPR4\#2 }}$

GPR4
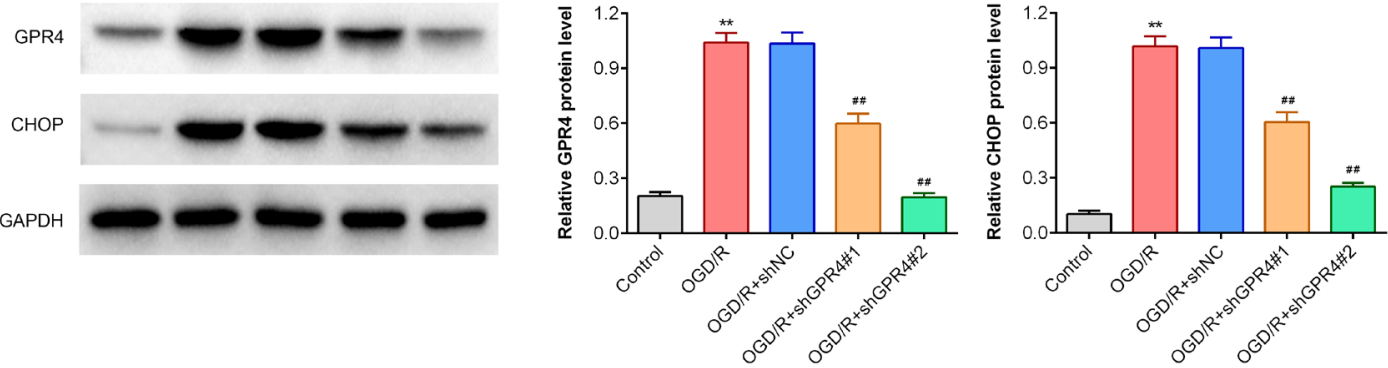

B

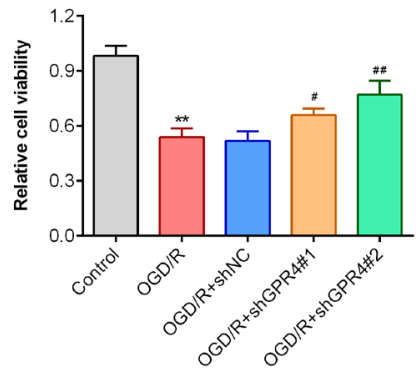

C

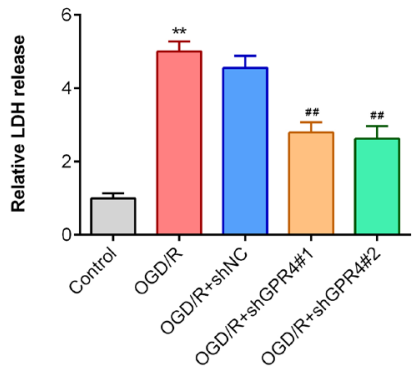

Figure 2. Knockdown of GPR4 reduces SH-SY5Y cell damage induced by OGD/R.

SH-SY5Y cells exposed to OGD/R were transfected with shRNA negative control (shNC) or shGPR4\#1 or shGPR4\#2 for $24 \mathrm{~h}$. (A) The protein levels of GPR4 and CHOP were measured by Western blotting $24 \mathrm{~h}$ after transfection. ${ }^{* *} p<0.01$ vs control group; $\#$ \# $p<0.01$ vs OGD/R group. (B) Cell viability was measured by MTT assay $24 \mathrm{~h}$ after transfection. ${ }^{* *} p<0.01$ vs control group; ${ }^{*} p<0.05,{ }^{\# *} p<0.01$ vs OGD/R group. (C) $\mathrm{LDH}$ release was measured $24 \mathrm{~h}$ after transfection. ${ }^{* *} p<0.01$ vs control group; ${ }^{\# p} p<0.01$ vs OGD/R group.

A
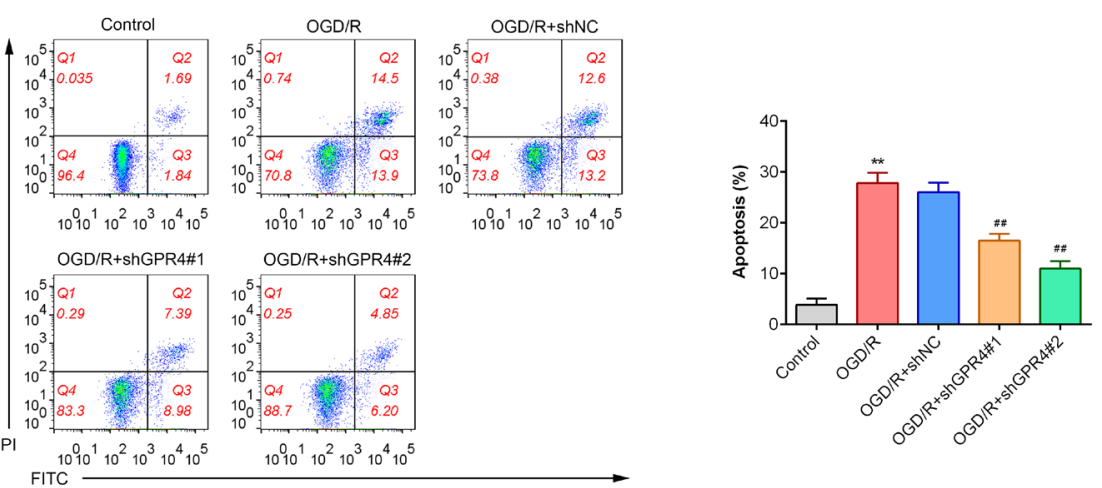

B

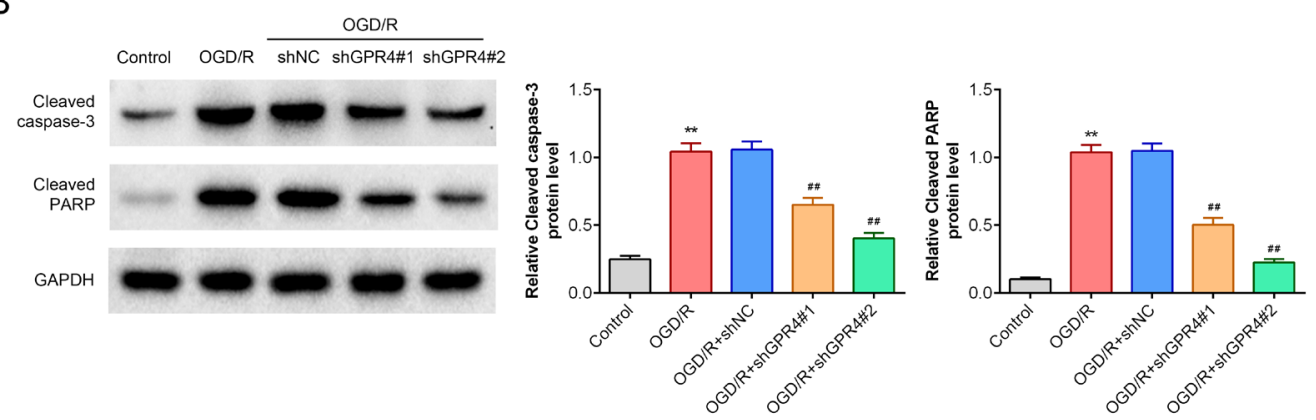

Figure 3. Knockdown of GPR4 reduces SH-SY5Y cells apoptosis induced by OGD/R.

SH-SY5Y cells exposed to OGD/R were transfected with shRNA negative control (shNC) or shGPR4\#1 or shGPR4\#2. (A) Apoptosis was measured by flow cytometry $24 \mathrm{~h}$ after transfection. ${ }^{* *} p<0.01 \mathrm{vs}$ control group; ${ }^{* \#} p<0.01 \mathrm{vs}$ OGD/R group. (B) The protein levels of cleaved caspase 3 and cleaved PARP were measured by Western blotting $24 \mathrm{~h}$ after transfection. ${ }^{* *} p<0.01$ vs control group; \# $p<0.01$ vs OGD/R group. 
A

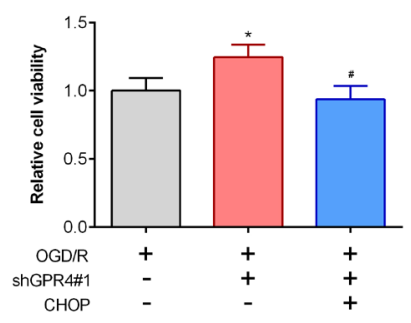

B

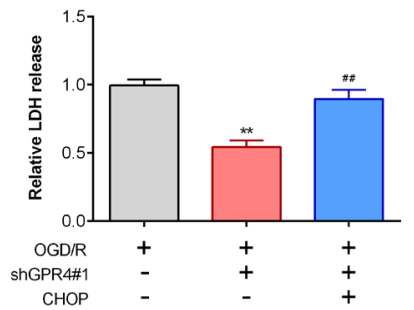

C
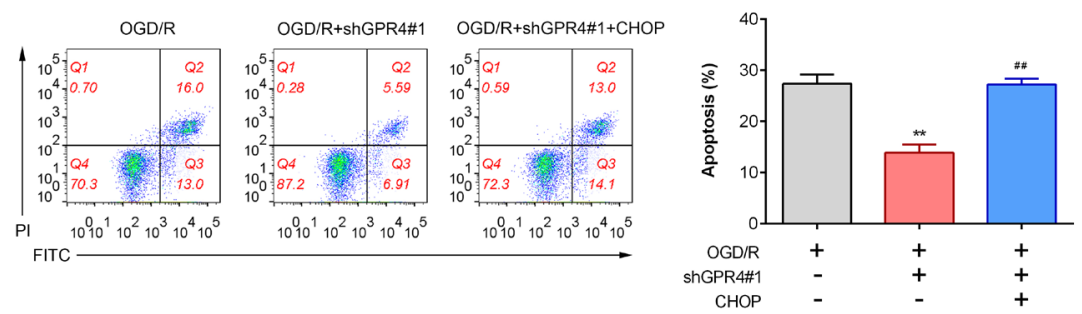

Figure 4. GPR4 promotes OGD/R-induced SH-SY5Y cells damage via CHOP.

SH-SY5Y cells exposed to OGD/R were transfected with shGPR4\#1 or infected with Ad-CHOP. (A) Cell viability was measured using MTT assay $24 \mathrm{~h}$ after transfection. ${ }^{*} p<0.05$ vs OGD/R group; ${ }^{*} p<0.05$ vs OGD/R + shGPR4\# 1 group. (B) LDH release was measured $24 \mathrm{~h}$ after transfection. ${ }^{* *} p<0.01$ vs OGD/R group; \#\# $p<0.01$ vs OGD/R + shGPR4\#1 group. C. Apoptosis was measured using flow cytometry $24 \mathrm{~h}$ after transfection. " $p<0.01$ vs OGD/R group; ${ }^{* \#} p<0.01$ vs OGD/R + shGPR4\#1 group.

pendent manner, indicating that the nerve injury induced by $\mathrm{OGD} / \mathrm{R}$ is related to the high expression of GPR4 and CHOP.

\section{Knockdown of GPR4 reduces nerve cell damage induced by $O G D / R$}

Surprisingly, we found that knockdown of GPR4 by two different shRNAs (shGPR4\#1 and shGPR4\#2) significantly reduced the levels of GPR4 and CHOP in SH-SY5Y cells exposed to OGD/R, and shGPR4\#2 performed better than shGPR4\#1 (Fig. 2A). In addition, we further investigated cell viability and $\mathrm{LDH}$ release. As shown in Fig. 2B, SH-SY5Y cells exposed to $\mathrm{OGD} / \mathrm{R}$ showed a significant decrease in cell viability, which was significantly increased after knockdown of GPR4 with shRNA (shGPR4\#1 and shGPR4\#2), and the performance of shGPR4\#2 was superior to shGPR4\#1. Conversely, LDH release in SH-SY5Y cells exposed to $\mathrm{OGD} / \mathrm{R}$ increased sharply compared to the control group, while LDH release was significantly reduced by knockdown of GPR4 with shRNA (shGPR4\#1 and shGPR4\#2), and the effect of shGPR4\#2 was comparable to that of shGPR4\#1. Taken together, these results indicated that knockdown of GPR4 reduces nerve cell damage induced by OGD/R.

\section{Knockdown of GPR4 reduces SH-SY5Y cells apoptosis induced by OGD/R}

In addition to exploring the effect of GPR4 on cell viability and $\mathrm{LDH}$ release, we also studied the effect of GPR4 on cell apoptosis. As shown in Fig. 3A, compared to the control group, the apoptosis rate of SH-SY5Y cells exposed to $\mathrm{OGD} / \mathrm{R}$ was significantly increased. However, after using different shRNAs to knock down GPR4 (shGPR4\#1 and shGPR4\#2), the apoptosis rate was significantly reduced. Further analysis showed that $\mathrm{OGD} / \mathrm{R}$ treatment promoted the expression of cleaved caspase 3 and cleaved PARP in SH-SY5Y cells while knocking down GPR4 prevented this process (Fig. 3B).
In summary, our results showed that knockdown of GPR4 reduces SH-SY5Y cells apoptosis induced by $\mathrm{OGD} / \mathrm{R}$.

\section{GPR4 promotes OGD/R-induced SH-SY5Y cell damage via CHOP}

To clarify the role of CHOP we overexpressed CHOP and investigated the effects on cell viability, $\mathrm{LDH}$ release and apoptosis. As shown in Fig. 4A, compared to the OGD/R group, after GPR4 was knocked down by shGPR4\#1, the viability of SH-SY5Y cells was significantly increased. It is worth noting that CHOP overexpression reversed the increase of cell viability caused by GPR4 knockdown (Fig. 4B and C). Moreover, CHOP overexpression also reversed the inhibitory effect of GPR4 knockdown on $\mathrm{LDH}$ release and apoptosis (Fig. 4B and $\mathrm{C}$ ). In summary, these results indicate that GPR4 promotes OGD/R-induced SH-SY5Y cells damage via CHOP.

\section{DISCUSSION}

Cerebral ischemia can cause sudden limitation of limb movement, language and sensory disorders, tissue damage and organ dysfunction, and even infarction of key brain regions (Zhang et al., 2019). In the process of rapid recovery of blood flow to the ischemic tissue, reperfusion injury occurs and causes secondary neuron injury (Ferlito et al., 2014; Caltagirone et al., 2016). The pathological process of CIRI involves many mechanisms. There is evidence that apoptosis plays an important role in the development of CIRI (Xiao et al., 2017; Zhao et al., 2018). In this study, the OGD/R model of SHSY5Y cells was established by subjecting SH-SY5Y cells to $\mathrm{OGD} / \mathrm{R}$. Our results showed that CIRI induced by $\mathrm{OGD} / \mathrm{R}$ caused decreased cell activity, increased LDH release, and increased apoptosis, which might be related to the overexpression of GPR4. Further experiments suggested that OGD/R-induced CIRI may involve endo- 
plasmic reticulum stress-related apoptosis (Yuqing Yang, 2020).

Endoplasmic reticulum (ER) is widely found in eukaryotic cells and is involved in lipid metabolism, steroid metabolism synthesis and calcium storage (Fabre et al., 2019). Endoplasmic reticulum stress (ERS) is a pathological state that causes the disorder of ER physiological function. CHOP gene plays an important role in the apoptosis induced by ERS (Lei et al., 2017). Under physiological conditions, CHOP expression is low in cells. During ERS response, the activation of IRE-1, PERK, and ATF6 induce the expression of CHOP, thus inducing apoptosis (Isodono et al., 2010). Studies showed that apoptosis induced by ERS is involved in CIRI (Liu et al., 2018). Pan et al., found that $\mathrm{OGD} / \mathrm{R}$ induced ERS and further promoted apoptosis in cerebral ischemia-reperfusion by increasing the expression of GRP78 and CHOP in SH-SY5Y cells (Pan et al., 2020). Similarly, in this study, we found that after ischemia, the expression of CHOP in cells increased significantly, which further reduced cell activity and increased LDH release and apoptosis. Cell viability was increased after lowering CHOP levels, while LDH release and apoptosis were significantly reduced, which was reversed by the forced overexpression of CHOP. Therefore, our results suggest that $\mathrm{CHOP}$ is involved in OGD/Rinduced CIRI, and its mechanism of action may be related to ERS-induced apoptosis.

Acidic $\mathrm{pH}$ can stimulate "proton-activated" $\mathrm{G}$ proteincoupled receptors (GPCRs, including GPR4, GPR68, GPR65, etc.) to transmit the relevant information (Yang et al., 2007; Sun et al., 2010). GPR4 was found to play a key role in HR/acidic-induced CHOP expression and apoptosis induction and is involved in endothelial cells adhesion and vascular endothelial cells inflammation (Chen et al., 2011). It was recently found that GPR4 expression is also increased in renal ischemia-reperfusion, and knockdown of GPR4 can improve renal ischemiareperfusion injury and inhibit apoptosis by inhibiting CHOP expression (Dong et al., 2017). Surprisingly, our study found that GPR4 also had a certain effect on CIRI. We found a high, time-dependent GPR4 expression in cells subjected to $\mathrm{OGD} / \mathrm{R}$, which reduced cell activity, increased LDH release, and increased apoptosis. After GPR4 depletion using shRNA, cell viability was increased, while LDH release and apoptosis were decreased. It is worth noting that the high expression of GPR4 was also accompanied by the high expression of CHOP, suggesting that the abnormal expression of GPR4 may be related to the apoptosis caused by ERS. More importantly, forced CHOP overexpression reversed the effects of GPR4 knockdown on cell viability, $\mathrm{LDH}$ release, and apoptosis. Taken together, our results suggest that CIRI is associated with high GPR4 expression and ERS-related apoptosis.

In conclusion, we investigated the role of GPR4 in cerebral ischemia-reperfusion and its underlying molecular mechanisms in vitro. It was found that ischemia-reperfusion decreased cell activity, increased $\mathrm{LDH}$ release and apoptosis, which may be related to the overexpression of GPR4 and CHOP. Collectively, our study provides a basis for further exploration of the mechanism of CIRI.

\section{Acknowledgements}

Not applicable.

\section{Funding}

Not applicable.

\section{Competing interests}

The authors state that there are no conflicts of interest to disclose.

\section{Ethics approval}

Not applicable.

\section{Statement of Informed Consent}

Not applicable.

\section{Availability of data and materials}

All data generated or analyzed during this study are included in this published article.

\section{Authors' contributions}

Chunli Xing designed the study, supervised the data collection, Guizhen Yan analyzed the data, interpreted the data, Qishuai Liu prepared the manuscript for publication and reviewed the draft of the manuscript. All authors have read and approved the manuscript.

\section{REFERENCE}

Caltagirone C, C Cisari, C Schievano, R Di Paola, M Cordaro, G Bruschetta, E Esposito,S Cuzzocrea (2016) Co-ultramicronized Palmitoylethanolamide/Luteolin in the Treatment of Cerebral Ischemia: from Rodent to Man. Transl Stroke Res 7: 54-69. https://doi. org/10.1007/s12975-015-0440-8

Chen A, Dong L, Leffler NR, Asch AS, Witte ON, Yang LV (2011) Activation of GPR4 by acidosis increases endothelial cell adhesion through the cAMP/Epac pathway. Plos One 6: e27586. https://doi. org/10.1371/journal.pone.0027586

Dong B, Zhang X, Fan Y, Cao S, Zhang X (2017) GPR4 knockout improves renal ischemia-reperfusion injury and inhibits apoptosis via suppressing the expression of CHOP. Biochem J 474: 4065-4074. https://doi.org/10.1042/bcj20170676

Dong B, Zhou H, Han C, Yao J, Xu L, Zhang M, Fu Y, Xia Q (2014) Ischemia/reperfusion-induced CHOP expression promotes apoptosis and impairs renal function recovery: the role of acidosis and GPR4. Plos One 9: e110944. https://doi.org/10.1371/journal. pone.0110944

Dong L, Krewson EA, Yang LV (2017) Acidosis activates endoplasmic reticulum stress pathways through GPR4 in human vascular endothelial cells. Int J Mol Sci 18. https://doi.org/10.3390/ ijms 18020278

Dong L, Li Z, Leffler NR, Asch AS, Chi JT, Yang LV (2013) Acidosis activation of the proton-sensing GPR4 receptor stimulates vascular endothelial cell inflammatory responses revealed by transcriptome analysis. Plos One 8: e61991. https://doi.org/10.1371/journal. pone.0061991

Fabre B, Livneh I, Ziv T, Ciechanover A (2019) Identification of proteins regulated by the proteasome following induction of endoplasmic reticulum stress. Biochem Biophys Res Commun 517: 188-192. https://doi.org/10.1016/j.bbrc.2019.07.040

Ferlito M, Wang Q, Fulton WB, Colombani PM, Marchionni L, FoxTalbot K, Paolocci N, Steenbergen C (2014) Hydrogen sulfide [corrected] increases survival during sepsis: protective effect of CHOP inhibition. I Immunol 192: 1806-1814. https://doi.org/10.4049/jimmunol.1300835

Hu H, Tian M, Ding C, Yu S (2018) The C/EBP homologous protein (CHOP) transcription factor functions in endoplasmic reticulum stress-induced apoptosis and microbial infection. Frontiers Immunol 9: 3083. https://doi.org/10.3389/fimmu.2018.03083

Hu YQ, Chen W, Yan MH, Lai JJ, Tang N, Wu L (2017) Ischemic preconditioning protects brain from ischemia/reperfusion injury by attenuating endoplasmic reticulum stress-induced apoptosis through PERK pathway. Eur Rev Med Pharmacol Sci 21: 5736-5744. https:// doi.org/10.26355/eurrev_201712_14020

Isodono K, Takahashi T, Imoto H, Nakanishi N, Ogata T, Asada S, Adachi A, Ueyama T, Oh H, Matsubara H (2010) PARM-1 is an endoplasmic reticulum molecule involved in endoplasmic reticulum stress-induced apoptosis in rat cardiac myocytes. Plos One 5: e9746. https://doi.org/10.1371/journal.pone.0009746

Lei Y, Wang S, Ren B, Wang J, Chen J, Lu J, Zhan S, Fu Y, Huang L, Tan J (2017) CHOP favors endoplasmic reticulum stress-induced apoptosis in hepatocellular carcinoma cells via inhibition of au- 
tophagy. Plos One 12: e0183680. https://doi.org/10.1371/journal. pone. 0183680

Liu C, Fu Q, Mu R, Wang F, Zhou C, Zhang L, Yu B, Zhang Y, Fang $\mathrm{T}$, Tian F (2018) Dexmedetomidine alleviates cerebral ischemia-reperfusion injury by inhibiting endoplasmic reticulum stress dependent apoptosis through the PERK-CHOP-Caspase-11 pathway. Brain Res 1701: 246-254. https://doi.org/10.1016/j.brainres.2018.09.007

Pan H, Zhao F, Yang Y, Chang N (2020) Overexpression of long noncoding RNA SNHG16 against cerebral ischemia-reperfusion injury through miR-106b-5p/LIMK1 axis. Life Sci 254: 117778. https:// doi.org/10.1016/j.lfs.2020.117778

Pfeilschifter W, Czech-Zechmeister B, Sujak M, Mirceska A, Koch A, Rami A, Steinmetz H, Foerch C, Huwiler A, Pfeilschifter J (2011) Activation of sphingosine kinase 2 is an endogenous protective mechanism in cerebral ischemia. Biochem Biophys Res Commun 413: 212-217. https://doi.org/10.1016/j.bbrc.2011.08.070

Shang W, Tang Z, Gao Y, Qi H, Su X, Zhang Y, Yang R (2017) LncRNA RNCR3 promotes Chop expression by sponging miR185-5p during MDSC differentiation. Oncotarget 8: 111754-111769. https://doi.org/10.18632/oncotarget.22906

Sun X, Yang LV, Tiegs BC, Arend LJ, McGraw DW, Penn RB, Petrovic S (2010) Deletion of the $\mathrm{pH}$ sensor GPR4 decreases renal acid excretion. $J$ Am Soc Nephrol 21: 1745-1755. https://doi. org/10.1681/asn.2009050477

Wu MY, Yiang GT, Liao WT, Tsai AP, Cheng YL, Cheng PW, Li CY, Li CJ (2018) Current mechanistic concepts in ischemia and reperfusion injury. Cell Physiol Biochem 46: 1650-1667. https://doi. org/10.1159/000489241

Xiao B, Chai Y, Lv S, Ye M, Wu M, Xie L, Fan Y, Zhu X, Gao Z (2017) Endothelial cell-derived exosomes protect SH-SY5Y nerve cells against ischemia/reperfusion injury. Int I Mol Med 40: 12011209. https://doi.org/10.3892/ijmm.2017.3106

Yang LV, Radu CG, Roy M, Lee S, McLaughlin J, Teitell MA, IruelaArispe ML, Witte ON (2007) Vascular abnormalities in mice deficient for the $G$ protein-coupled receptor GPR4 that functions as a pH sensor. Mol Cell Biol 27: 1334-1347. https://doi.org/10.1128/ mcb.01909-06

Song F, Zhan H, Liang Y, He X, Guo L (2020) Lapatinib promotes ovarian cancer cell apoptosis through mROS-HtrA2/Omi pathways. Eur J Gynaecol Oncol 41: 598-603. https://doi.org/10.31083/j. ejgo.2020.04.5247

Zhang Z, Liu S, Huang S (2019) Thymosin $\beta 4$ prevents oxygen-glucose deprivation/reperfusion-induced injury in rat cortical neurons. Neuropsych Dis Treat 15: 2385-2393. https://doi.org/10.2147/NDT. S208600

Zhao JJ, Xiao H, Zhao WB, Zhang XP, Xiang Y, Ye ZJ, Mo MM, Peng XT, Wei L (2018) Remote ischemic postconditioning for ischemic stroke: a systematic review and meta-analysis of randomized controlled trials. Chin Med J (Engl) 131: 956-965. https://doi. org/10.4103/0366-6999.229892

Zhao Q, Wang X, Chen A, Cheng X, Zhang G, Sun J, Zhao Y, Huang Y, Zhu Y (2018) Rhein protects against cerebral ischemic-/reperfusion-induced oxidative stress and apoptosis in rats. Int J Mol Med 41: 2802-2812. https://doi.org/10.3892/ijmm.2018.3488

Zhou AX, Wang X, Lin CS, Han J, Yong J, Nadolski MJ, Borén J, Kaufman RJ, Tabas I (2015) C/EBP-Homologous Protein (CHOP) in vascular smooth muscle cells regulates their proliferation in aortic explants and atherosclerotic lesions. Circ Res 116: 1736-1743. https://doi.org/10.1161/circresaha.116.305602 